\title{
Global Machine-to-Machine Sensor Network for Wide Area Healthcare Service
}

\author{
Sang-Joong Jung ${ }^{1}$, Anssi Makynen ${ }^{2}$, Risto Myllyla ${ }^{2}$, Wan-Young Chung ${ }^{1}$ \\ ${ }^{1}$ Department of Electronic Engineering, Pukyong National University, Busan 608-737, Korea \\ ${ }^{2}$ Department of Electrical Engineering, University of Oulu, P.O. Box 4500, Oulu 90014, Finland \\ wychung@pknu.ac.kr
}

\begin{abstract}
:
The global machine-to-machine (M2M) network is proposed to support wide area healthcare service using the concept of IPv6 over low power wireless personal area (6LoWPAN). This network can give international healthcare services between countries and intercity healthcare services between cities with the excellent accessibility in a hierarchical network structure. Low power embedded wearable sensors are designed to measure health parameters dynamically and connected to the M2M node for wireless transmission through the internet or external IP enable networks via the M2M gateway. Practical tests were conducted by the M2M gateway with the effective use of IEEE 802.15.4 and the 6LoWPAN protocol in wide area. The physical health state is estimated by the heart rate variability analysis in time and frequency domain with the significant information of autonomic nervous system in the server. Our approach for the global M2M network is managed to process the large amount of biomedical signals through the 6LoWPAN and internet accurately. Patients may comfortably monitor their own health condition regardless of the place in the global M2M network environment.
\end{abstract}

Key words: machine-to-machine, healthcare service, IPv6 over low power wireless personal area network, health condition, heart rate variability, and autonomic nervous system

\section{Introduction}

The machine-to-machine (M2M) is a main form of internet of things (loT) at the present stage. This decade is widely predicted to see the rise of M2M communications over wired and wireless links [1]. The origin of M2M communications was cloudy because of the many different possibilities of its inception, when cellular technology first began to learn to connect directly to other computer systems. With the dramatic penetration of embedded devices, M2M communications become a dominant communication paradigm in many applications which concentrate on data exchanging among the machines to make smart up as a narrow sense and currently networked applications and services, whose core is intelligence interacting of machines as a general sense [2][3].

As a prime benefit of these new strategies, IPbased wireless networks are the catalyst of the accelerated M2M services innovation that will help them identify hidden growth opportunities and take place in M2M services. The evolution of M2M has begun in developing a wireless sensor network with the help of IPv6 technique [4]. Advances in the M2M network allow the establishment of wireless sensor network by the efficient addressing mechanism of IPv6 over IEEE 802.15.4 standard to every node to enhance the quality of data transmission and extend healthcare service coverage. Therefore, the implementation of the global M2M network into healthcare application can replace traditional healthcare system based on wireless sensor network and give new advantages and benefits with a global IPv6 address for the patients such as easiness of measurement, extension of network, accessibility, and reliability as the beginning of a new generation. The proposed system can be applicable to improve the expansion of healthcare service coverage with the efficient support of IPv6 over low power wireless personal area network (6LoWPAN) in wide areas [5].

\section{System Design}

The overall architecture of global M2M network to evaluate patient's health condition according to the requirements of the 6LoWPAN is shown in Fig. 1. As the core hardware in this system, the M2M devices are designed to measure and transmit the electrocardiogram (ECG) and photoplethysmogram (PPG) for the flexible and scalable M2M network as shown in Table 1. 


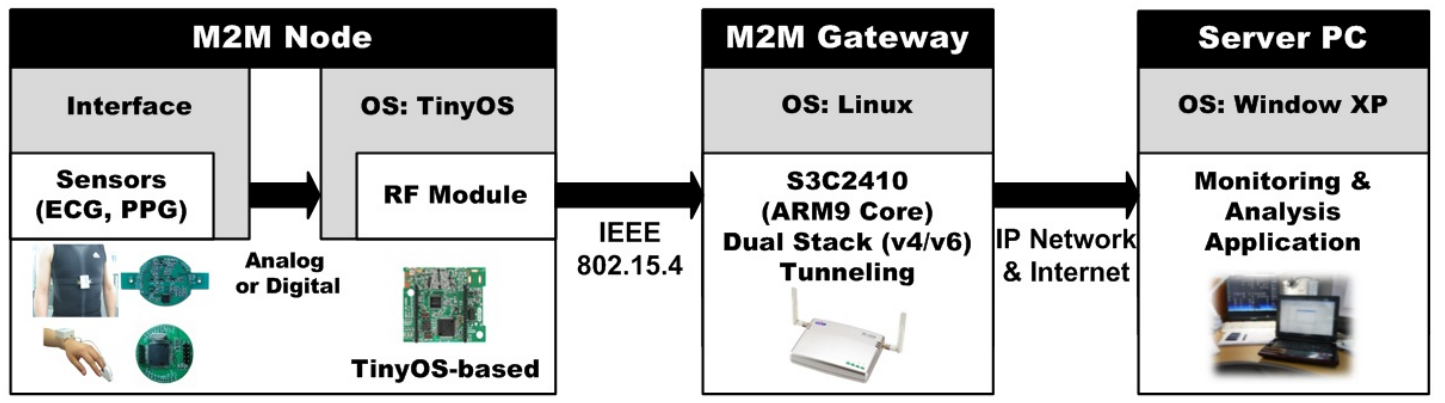

Fig. 1. Block diagram of overall system architecture.

Tab. 1: Specifications of M2M devices

\begin{tabular}{|c|c|c|}
\hline Module & Items & Descriptions \\
\hline $\begin{array}{l}\text { ECG } \\
\text { Sensor }\end{array}$ & $\begin{array}{l}\text { Electrodes } \\
\text { Gain } \\
\text { Cut-off Freq. } \\
\text { Power }\end{array}$ & $\begin{array}{l}\text { Strap (Polar ) } \\
500(27 \mathrm{~dB}) \\
0.5-34 \mathrm{~Hz} \\
3.3 \mathrm{~V}\end{array}$ \\
\hline $\begin{array}{l}\text { PPG } \\
\text { Sensor }\end{array}$ & $\begin{array}{l}\text { LED } \\
\text { Gain } \\
\text { Cut-off Freq. } \\
\text { Power }\end{array}$ & $\begin{array}{l}940 \mathrm{~nm} \text { Infrared } \\
100(20 \mathrm{~dB}) \\
0.5-10 \mathrm{~Hz} \\
3.3 \mathrm{~V}\end{array}$ \\
\hline $\begin{array}{l}\text { Sensor } \\
\text { Node }\end{array}$ & $\begin{array}{l}\text { MCU } \\
\text { RF } \\
\text { Transceiver } \\
\text { OS } \\
\text { Power }\end{array}$ & $\begin{array}{l}\text { MSP430 (16 bit) } \\
\text { IEEE } 802.15 .4 \\
\text { TI CC2420 } \\
\text { TinyOS } 2 . x \\
\text { AC } 220 \text { V-DC } 3 \text { V }\end{array}$ \\
\hline $\begin{array}{c}\text { M2M } \\
\text { Gateway }\end{array}$ & $\begin{array}{l}\text { CPU } \\
\text { Network } \\
\text { Transceiver } \\
\text { I/O Interface } \\
\text { Power }\end{array}$ & $\begin{array}{l}\text { S3C2410 (ARM9) } \\
802.3 \text { (10 Mbps) } \\
802.11 \mathrm{~b} / \mathrm{g} \text { (WLAN) } \\
\text { TI CC2420 } \\
\text { RS-232, USB2.0 } \\
\text { DC } 5 \mathrm{~V}\end{array}$ \\
\hline
\end{tabular}

The ECG sensor is designed by two conductive fabric electrodes with the size of $8 \mathrm{~cm}$ from inner chest part of shirt and the PPG sensor is also designed by the reflectance probe from finger [6]. The M2M nodes connected to the wearable sensors are placed on patient's body and are mainly responsible for collecting and transmitting the sampled signals at $100 \mathrm{~Hz}$ for the ECG and $75 \mathrm{~Hz}$ for the PPG to the M2M gateway. In particular, the 6LoWPAN protocol stack is implemented on top of IEEE 802.15.4 layer in the M2M nodes operated to transmit packets in higher-level protocol which is the 6LoWPAN ad hoc on-demand distance vector (LOAD) routing protocols developed by IETF group. In the 6LoWPAN, LOAD routing protocol can guarantee flexibility from various changes of topologies in quickly operating wireless sensor network. The M2M gateway is placed between two different networks which are the
IPV6 over IEEE 802.15.4 on one side and the IP network on the other. Moreover the M2M gateway performs the global address translation to either 16-bit short address or IEEE EUI64-bit extended address [7]. TinyOSbased M2M node is allocated with its own IP address from the M2M gateway over IPv6 packets. First of all, the IPv6 address must be defined at the M2M gateway and server PC for the IPV6 communication. As the IP network can be generally accessed by the IPv4 address, the IPv6 to IPv4 tunneling process which changes address format in the M2M gateway is required to approach the server PC through the internet. In this experiment, the IPv6 address, as a 2002:cbfa:79cf::cbfa:79cf from IPv4 address of 203.250.121.204 (Pukyong National University, Korea), is assigned to register at the server PC. The M2M gateway is also assigned with a IPv6 address of 2002:82e7:3b66::1 (University of Oulu, Finland) to allocate lower prefix address to the connected the M2M nodes as a 2002:82e7:3b66:100:22:ff:fe00:1 in the 6LoWPAN by auto-configuration function as shown in Table 2.

Tab. 2: Assignment of IP addresses

\begin{tabular}{|c|l|}
\hline Items & \multicolumn{1}{|c|}{ Assigned IP Address } \\
\hline Server PC & IPv4: 203.250.121.207 (Korea) \\
& IPv6: 2002:cbfa:79cf::cbfa:79cf \\
\hline $\begin{array}{c}\text { M2M } \\
\text { Gateway }\end{array}$ & IPv4: 130.231.59.102 (Finland) \\
IPv6: 2002:82e7:3b66::1 \\
\hline M2M Node & Prefix IPv6: \\
& 2002:82e7:3b66:100:22:ff:fe00:1 \\
\hline
\end{tabular}

\section{Experimental Results}

The practical tests were conducted to observe the performance of the global M2M network in real time as shown in Fig. 2. It performs an accurate recognition of position with IP to support the healthcare services even if the patients are under unconscious situation. 


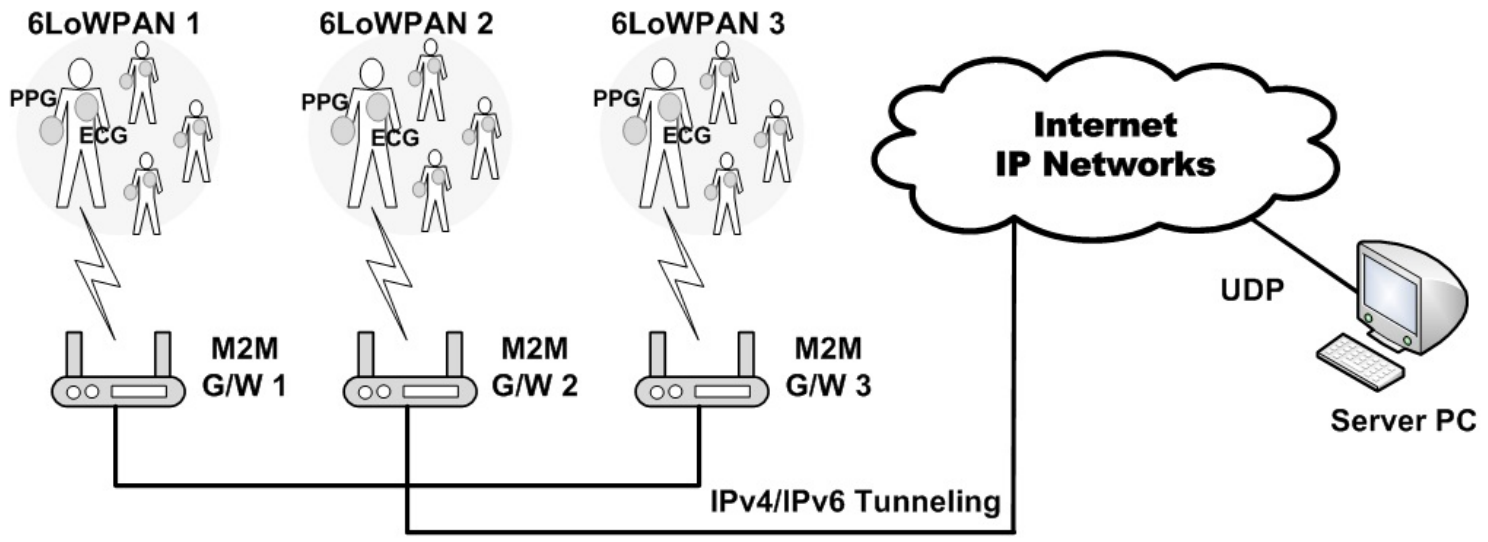

Fig. 2. Practical tests of the global M2M network in wide area.

The M2M node is connected to the wearable sensors that placed on the patient's body which collect the health parameters and further transfer the data to the M2M gateway. The M2M node stores the measured signals in buffer where the signals used up of 25 bytes of data packets respectively. The total 29 bytes of 6 LoWPAN packet except the header packet are included in payload with the 2 bytes of node ID, 2 bytes of sensor type, and 25 bytes of data. The M2M gateway is the medium between the 6LoWPAN and internet that delivers the packets of the ECG and PPG signals. In receipt of the all transmitted packets via user datagram protocol (UDP) communication in the server, the health monitoring program verifies the records of sensing values and the analysis results of relevant personal information in detail dynamically.

To support the healthcare service, the heart rate variability (HRV) analysis in time and frequency domains extracted from the ECG or PPG signal is defined as the constant changes of the interval between the heart rates (HR) to effectively react patient's health condition [8]. In addition, the HRV is regulated predominantly by the autonomic nervous system (ANS) as the certain individual's abilities indicator which describes the nerves that are concerned with regulation of bodily functions without volition or consciousness. The ANS is influenced from sympathetic and parasympathetic nerves that sympathetic nerves excite the heart, increasing the $\mathrm{HR}$, and parasympathetic nerves reduce the $H R$ [9]. By accurately measuring the HRV signals, the detected variation can be used to decide the psychological and physiological stress and fatigue on the body. Table 3 is summarized for the comparison of mean values of HRV characteristics in time and frequency domain. Two test subjects (male, 28 and male, 32) without any heart diseases were recruited for 10 minutes short term test and an hour long term test.
Tab. 3: HRV analysis performance for health condition evaluation

\begin{tabular}{|c|c|c|}
\hline \multicolumn{3}{|c|}{ Time Domain } \\
\hline Parameters & Normal & Stressed \\
\hline HR (bpm) & $78.2 \pm 1.49$ & $73.2 \pm 0.14$ \\
\hline HRV $(\mathrm{ms})$ & $769.2 \pm 28.6$ & $821.9 \pm 13.3$ \\
\hline SDNN $(\mathrm{ms})$ & $47.2 \pm 4.2$ & $33.5 \pm 3.9$ \\
\hline RMSSD $(\mathrm{ms})$ & $38.3 \pm 3.8$ & $23.9 \pm 2.9$ \\
\hline \multicolumn{3}{|c|}{ Frequency Domain } \\
\hline Parameters & Normal & Stressed \\
\hline LF $\left(\mathrm{ms}^{2}\right)$ & $319.98 \pm 26.95$ & $246.42 \pm 11.27$ \\
\hline HF $\left(\mathrm{ms}^{2}\right)$ & $128.43 \pm 18.30$ & $70.72 \pm 21.68$ \\
\hline Ratio of LF/HF & $2.49 \pm 0.11$ & $3.63 \pm 0.95$ \\
\hline
\end{tabular}

For the analysis of HRV signals in time domain, the parameters of mean HR, standard deviation normal to normal (SDNN), and root mean square of successive differences (RMSSD) are interpreted to estimate health condition such as normal and stressed states. Subjects were asked to overwork and feel drowsy for the stressed state. The correlation of SDNN and RMSSD are significantly verified to explain the different pattern between the normal and stressed states. The mean values of SDNN and RMSSD are indicated as $47.2 \mathrm{~ms}$ of the SDNN and $38.3 \mathrm{~ms}$ of the RMSSD in normal state in the relaxed situation during the experiment time period. On the other hands, under stressed state, the mean values of SDNN and RMSSD are indicated as $33.5 \mathrm{~ms}$ of SDNN and $23.9 \mathrm{~ms}$ of RMSSD with the clearly lower values. From the time domain analysis, the quite uniform and stable HRV signals are extracted by the activation of parasympathetic nerve in the stressed state. On the contrary, the unstable HRV signals which mean that our body is always ready to work against external interrupt are extracted in the normal state. 
Frequency domain analysis shows the power spectrum density (PSD) method on how power distributes as a function of frequency accordingly illustrates the likeliness of stress emotions. Three main spectral components are required for spectrum power indicator analysis: very low frequency (VLF), low frequency (LF), and high frequency (HF) components which are usually made from PSD analysis in absolute values of power. The representation of LF and HF power components emphasizes the controlled and balanced behavior of the two branches of the ANS and be quoted with the absolute values of LF and HF power components in total. The mean values of HF and LF power components are somewhat different between the normal and stressed state as shown in Table 3. Based on these results, the ratio of LF/HF was used to evaluate patient's health condition from the normal state $(R=2.49)$ and the stressed state $(R=3.63)$.

In this work, for the possible indicator and reliable estimation of patient's health condition, the criteria ranges of ANS balance are introduced and confirmed by the results of HRV analysis. As getting stressed, the mean values of RMSSD, LF, and HF show a decreasing trend, while the mean value of ratios of LF/HF shows an increasing trend. These results describe that the mean values of RMSSD, LF, $\mathrm{HF}$, and ratio of LF/HF can be used as an indicator for presenting ANS balance to classify health condition. The ratio of LF/HF, RMSSD, LF, HF, and features of sympathetic and parasympathetic nerves are verified to define a criteria range of ANS balance for estimation of health condition.

Tab. 4: Criteria ranges of ANS balance for estimation of health condition

\begin{tabular}{|c|c|c|}
\hline Parameters & Normal & Stressed \\
\hline RMSSD $(\mathrm{ms})$ & $32-50$ & $14-32$ \\
\hline LF $\left(\mathrm{ms}^{2}\right)$ & $280-330$ & $220-280$ \\
\hline HF $\left(\mathrm{ms}^{2}\right)$ & $80-140$ & $40-80$ \\
\hline Ratio of LF/HF & $2-3$ & $3-4$ \\
\hline Sympathetic & Normal & Inactivation \\
\hline Parasympathetic & Normal & Activation \\
\hline
\end{tabular}

\section{Conclusions}

A global M2M network for international or intercity healthcare service is successfully implemented based on the 6LoWPAN. The M2M devices are designed and used for the measurement of ECG and PPG signals and transmission purpose to the server PC through the internet under the different local coverage areas. By combining the 6LoWPAN and IP network using IPv6 address, the extreme extension of network and the higher accessibility of M2M node are realized. In addition, the HRV signals are used to estimate patient's health condition by criteria ranges of ANS balance for estimation of health condition. This paper proposes the key ideas of establishing the global M2M network and the efficient support of IPv6 over IEEE 802.15.4 protocol in healthcare application.

\section{Acknowledgments}

The research was financially supported by the Ministry of Education, Science Technology (MEST) and National Research Foundation of Korea (NRF) through the Human Resource Training Project for Regional Innovation.

\section{References}

[1] G. Lawton, Machine-to-Machine Technology Gears Up for Growth, Computer 37, 12-15 (2004); doi: 10.1109/MC.2004.137

[2] S. Whitehead, Adopting Wireless Machine-toMachine Technology, IEEE Computing and Control Engineering Journal 15(5), 40-46 (2004); doi: $10.1049 /$ cce:20040509

[3] C. Inhyok, Y. Shah, A.U. Schmidt, A. Leicher, M.V. Meyerstein, Trust in M2M Communication, IEEE Vehicular Technology Magazine 4(3), 69-75 (2009); doi: 10.1109/MVT.2009.933478

[4] Z. Shelby, C. Bormann, 6LoWPAN: The Wireless Embedded Internet, Wiley (2009)

[5] Internet Engineering Task Force, http://www.ietf.org/

[6] S. J. Jung, T. H. Kwon, W. Y. Chung, A New Approach to Design Ambient Sensor Network for Real Time Healthcare Monitoring System, Proceedings of IEEE Sensors 2009 Conference (2009), pp. 576-580; doi: 10.1109/ICSENS.2009.5398310

[7] S. J. Jung, W. Y. Chung, Flexible and Scalable Patient's Health Monitoring System in 6LoWPAN, Sensor Letters 9, 778-785 (2011); doi:10.1166/sl.2011.1614

[8] M. Malik, Heart Rate Variability: Standards of Measurement, Physiological Interpretation, and Clinical Use, Circulation 93, 1043-1065 (1996); doi: 10.1161/01.CIR.93.5.1043

[9] U. Dulleck, A. Ristl, M. Schaffner, B. Torgler, Heart rate variability, the autonomic nervous system, and neuroeconomic experiments, Journal of Neuroscience Psychology and Economics 4, 117-124 (2011); doi: 10.1037/a0022245 